\title{
Idiopathic Pulmonary Hemosiderosis: A Case Report with Review of Related Literature
}

\section{Gauri S Shah ${ }^{1 *}$, Prince Parakh ${ }^{1}$ Shiva Chalise ${ }^{1}$, Kayur Mehta1 and Om P Mishra ${ }^{2}$}

${ }^{1}$ Department of Pediatrics and Adolescent Medicine, B.P. Koirala Institute of Health Sciences, Dharan, Nepal ${ }^{2}$ Department of Pediatrics, Institute of Medical Sciences, Banaras Hindu University, Varanasi, India

\begin{abstract}
Pulmonary hemosiderosis is an uncommon disorder in pediatric population in developing countries. It may affect the lung in an isolated form of Idiopathic Pulmonary Hemosiderosis (IPH) or as a manifestation of systemic disorders. The present patient belonging to Mongolian race presented with history of fever, cough, respiratory distress and previous episode of hemoptysis. The child had anemia, negative tuberculin test and no acid-fast bacilli in sputum and presence of bilateral pulmonary infiltrates in X-ray chest. Sputum examination showed iron-laden macrophages and no secondary cause could be ascertained. The patient responded well to prednisolone treatment alone. Idiopathic pulmonary hemosiderosis can be diagnosed based on the clinical features and sputum examination. Lung biopsy and high resolution CT thorax may not be undertaken in setting where facilities are not available.
\end{abstract}

Keywords: Pulmonary hemosiderosis; Sputum examination; Children

\section{Introduction}

Virchow first described Idiopathic Pulmonary Hemosiderosis (IPH) in 1864 as "brown" lung indurations [1]. It is a disorder of unknown etiology that is characterized by recurrent or chronic hemorrhage in the alveoli and accumulation of hemosiderin pigment in the lungs. Clinically, it manifests as a triad of hemoptysis, iron deficiency anemia and diffuse parenchymal infiltrates in chest radiograph [2]. Its course is extremely variable and most of the patients continue to have episodes of pulmonary hemorrhages despite therapy. Diagnosis is usually based on sputum examination, lung biopsy and high resolution CT thorax. Various immunosuppressive drugs such as prednisolone [3], hydroxychloroquine [4] and cycophosphamide [5] have been tried to improve the survival of these patients. However, lung biopsy and CT scan cannot be performed in this case as these facilities are not available at every center in a developing country like Nepal. We here report a patient that was diagnosed based on the clinical symptoms, sputum microscopy, X-ray chest findings and good response to prednisolone therapy.

\section{Case Presentation}

A 9year old Mongolian female child was admitted to the Department of Pediatrics with chief complaints of fever, cough, fast breathing and chest pain for 3 days. Fever was sudden in onset, continuous, recorded up to $103^{\circ} \mathrm{F}$ and not associated with chills and rigors. Cough was dry in nature, intermittent and there was no postural variation. Fast breathing was not associated with chest indrawing, altered sensorium or decreased activity. Chest pain was occasionally present and it was on the right side. There was no history of foreign body inhalation, seizures, cyanosis, and loss of consciousness, palpitation, orthopnea, and paroxysmal nocturnal dyspnoea, swelling of body, decreased urine output, joint pain and skin rashes. The child had an episode of hemoptysis and blood transfusion in the past.

On examination, she was conscious, oriented but tachypneic. Her blood pressure was $110 / 60 \mathrm{~mm} \mathrm{Hg}$, pulse-126/minute, respiration rate $-46 / \mathrm{min}$ and temperature was $100^{\circ} \mathrm{F}$. Child had moderate pallor but no lymphadenopathy, clubbing and cyanosis. On anthropometry examination her weight was $14 \mathrm{~kg}$, height was $115 \mathrm{~cm}$ and body mass index was 10.7. All the parameters were less than $3 \%$ for her age; indicating that she had growth retardation also. On examination of respiratory system, there were crepitations on right infrascapular, interscapular regions and also on the left hemi thorax. Cardiovascular system revealed normal first and second heart sounds and haemic murmur at pulmonary area. On examination of abdomen, it was soft, non tender, no distension and liver was palpable $4 \mathrm{~cm}$ below the right costal margin with a span of $8 \mathrm{~cm}$ and the spleen was $3 \mathrm{~cm}$ in spinoumbilical line. There was no ascites. Central nervous system was normal.

Investigations showed total leucocyte count of 6,500 per cubic millimeter out of which $60 \%$ were neutrophils, $35 \%$ were lymphocytes, $5 \%$ were eosinophils and the hemoglobin was $7 \mathrm{~g} / \mathrm{dl}$. The general blood picture was microcytic-hypochromic. Tuberculin test was negative and X-ray chest showed bilateral infiltrates (Figure 1). Sputum cytology showed predominantly hemosiderin laden macrophages, few lymphocytes and squamous epithelial cells with occasional columnar cells and there were no malignant cells. Acid-fast bacilli staining of sputum was negative. The antinuclear antibody, ANCA, antiDs DNA antibodies were negative. Renal function tests were normal.

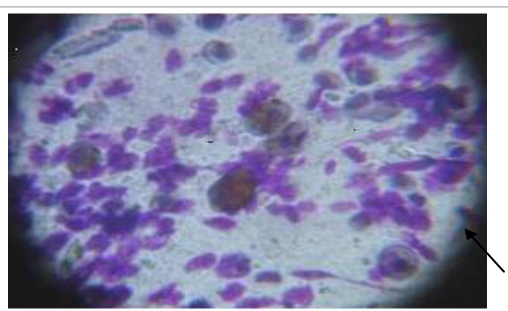

Figure 1: Microscopic examination of sputum: Iron- laden macrophages were Seen ( $\mathrm{H} \times \mathrm{E}$ stain, shown by arrow).

*Corresponding author: Gauri S Shah, Head of the Department of Pediatrics and Adolescent Medicine, B.P. Koirala Institute of Health Sciences, Dharan, Nepal, Tel: +977-9842415770; Fax: +977-25528757; E-mail: gaurishankarshah@live.com

Received July 06, 2012; Accepted August 21, 2012; Published August 23, 2012

Citation: Shah GS, Parakh P, Chalise S, Mehta K, Mishra OP (2012) Idiopathic Pulmonary Hemosiderosis: A Case Report with Review of Related Literature. J Clin Case Rep 2:185. doi:10.4172/2165-7920.1000185

Copyright: $\odot 2012$ Shah GS, et al. This is an open-access article distributed under the terms of the Creative Commons Attribution License, which permits unrestricted use, distribution, and reproduction in any medium, provided the original author and source are credited. 


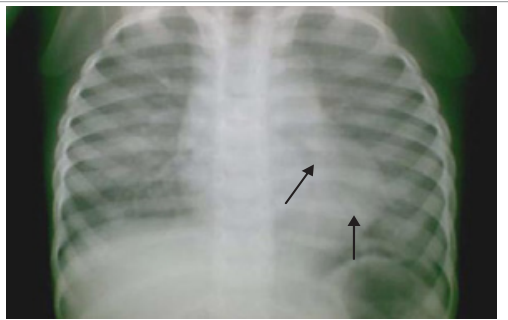

Figure 2: X-ray chest: Showing bilateral pulmonary infiltrates (Shown by arrow).

As this child had history of hemoptysis, anemia, infiltrates in the chest, and positive sputum for hemosiderin pigment, the diagnosis of pulmonary hemosiderosis was made. The child was managed with intravenous maintenance fluid, oxygen inhalation, ceftriaxone (100 $\mathrm{mg} / \mathrm{kg} /$ day in 2 divided doses), and packed red blood cell transfusion. After a week, child became stable and prednisolone was started by 2 $\mathrm{mg} / \mathrm{kg} /$ day for 2 weeks, then $1 \mathrm{mg} / \mathrm{kg} /$ day for 2 weeks and $1 \mathrm{mg} / \mathrm{kg} /$ dose on alternate days and continued till 12 weeks. In addition, oral iron and folic acid were also given. Anemia was corrected and patient did not have further episode of respiratory distress and hemoptysis.

\section{Discussion}

In developing country, when a patient presents with fever, anemia, hemoptysis and pulmonary infiltrates, the diagnosis of tuberculosis is often considered. But the present case had negative tuberculin test, absent acid-fast bacilli in sputum and positive sputum hemosiderinladen macrophages; indicative of pulmonary hemosiderosis. Bronchoalveolar lavage fluid microscopy, lung biopsy and CT chest could not be done due to lack of facilities at our centre. Although lung biopsy remains the gold standard test for the diagnosis, sputum examination for pigment deposition also offers an alternative approach in the diagnosis of the disease in a poor resource setting.

Zhang et al. [3] reported that the disease is because of immune imbalance and corticosteroid has good results. Saeed et al. [2] found that average survival of these patients was 2.5 years with overall 5 year survival of $86 \%$. Besides prednisolone, hydroxychloroquine [4] and cyclophosphamide [5] have been used in pediatric IPH with variable results. With the use of prednisolone in our case, anemia and pulmonary infiltrates (Figure 2) disappeared in the follow-up. Mortality can occur suddenly from acute pulmonary hemorrhage or after progressive pulmonary insufficiency due to pulmonary fibrosis. High-resolution thoracic computed tomography scans are useful for early detection of pulmonary fibrosis. Therefore, early definitive diagnosis and aggressive immunosuppressive therapy of IPH are imperative in order to avoid pulmonary fibrosis and mortality in these patients [6]. Our patient responded well to prednisolone treatment. However, long-term follow up is required to know the outcome in such patients.

\section{Conclusion}

Thus, it is evident that when a child presents with hemoptysis, pulmonary infiltrates in X-ray chest, negative tuberculin test and positive sputum for hemosiderin-loaded macrophages, one should think about pulmonary hemosiderosis in pediatric population. The steroid remains the sheet anchor of therapy.

\section{References}

1. Cordeiro M (1952) A case of idiopathic pulmonary hemosiderosis Quéri by splenectomy. Helv Paediatr Acta 7: 1-507.

2. Saeed MM, Woo MS, Mc Laughlin EF, Margetis MF, Keens TG (1999) Prognosis in idiopathic pediatric hemosiderosis. Chest 116: 721-725.

3. Zhang X, Wang L, Lu A, Zhang M (2010) Clinical Study of 28 Cases of paediatric idiopathic pulmonary haemosiderosis. J Trop Pediatr 56: 386-390.

4. Keller C, Langstan C (2000) Childhood idiopathic pulmonary hemosiderosis. N Engl J Med 343: 781.

5. Huang SH, Lee PY, Niu CK (2003) Treatment of pediatric idiopathic pulmonary hemosiderosis with low-dose cyclophosphamide. Ann Pharmacother 11: 16181621.

6. Yao TC, Huang IJ, Wong KS,Huang JL, Niu CK (2003) Idiopathic pulmonary haemosiderosis: An Oriental experience. J Paediatr Child Health 39: 27-30. 